\title{
Segmentation of Sputum Color Image for Lung Cancer Diagnosis Based on Neural Networks
}

\author{
Sammouda Rachid ${ }^{1}$, Noboru Niki ${ }^{1}$, Hiromu Nishitani ${ }^{2}$, \\ S. Nakamura ${ }^{3}$, and S. Mori ${ }^{3}$ \\ ${ }^{1}$ Dept. of Optical Science, University of Tokushima, Japan. \\ 2 Medical School of Tokushima, Japan. \\ 3 Tokushima Health Screening Center, Japan.
}

\begin{abstract}
The paper presents a method for automatic segmentation of sputum cells with color images, to develop an efficient algorithm for lung cancer diagnosis based on a Hopfield neural network. We formulate the segmentation problem as a minimization of an energy function constructed with two terms, the cost-term as a sum of squared errors, and the second term a temporary noise added to the network as an excitation to escape certain local minima with the result of being closer to the global minimum. To increase the accuracy in segmenting the regions of interest, a preclassification technique is used to extract the sputum cell regions within the color image and remove those of the debris cells. The former is then given with the raw image to the input of Hopfield neural network to make a crisp segmentation by assigning each pixel to label such as Background, Cytoplasm, and Nucleus. The proposed technique has yielded correct segmentation of complex scene of sputum prepared by ordinary manual staining method in most of the tested images selected from our database containing thousands of sputum color images.
\end{abstract}

Keywords. Sputum cells, Lung cancer diagnosis, RGB images, Segmentation, Optimization, Hopfield neural network.

\section{Introduction}

Lung cancer is nowadays becoming a serious disease in the world causing a huge number of deaths, this is because it is difficult to detect it in its early stage and possibly stop its danger. Several techniques are being used for scanning the lung area, such as X-ray Helical CT technique [1-3]. However, the analysis process of the three dimensional images obtained by this technique is still suffering from some limitations caused by the scanning process[4]. Recently, some medical researchers have proven that the analysis of sputum cells can assist for a successful diagnosis of lung cancer. For this reason, we attempt to make an automatic diagnostic system of lung cancer based on the analysis of the sputum images. In order to formulate general diagnostic rules, we have collected a database with thousands of sputum color images from patient and normal subjects. As a first step, we have developed a technique for unsupervised segmentation of the sputum color image. In this paper, we present a segmentation method of sputum color images prepared by the standard staining method proposed in [5], or a modification thereof. The Papanicolaou stain consists of several staining dyes, resulting in darkly stained nuclei but also in clearly visible 
cytoplasm, some appearing blue and others red. In the computer memory, each image is represented as three separate pixel matrices corresponding to red, green, and blue intensity ( $R, G$, and $B$ image planes). The segmentation is performed based on a preprocessing for the extraction of the regions of interest (ROI), and on an energy function minimization using a Hopfield neural network (HNN). We present results of the segmentation of simple and complex sputum images. The paper concludes with some discussion and comments and a brief summary of our future work.

\section{Hopfield Neural Network}

Hopfield neural network for the optimization application consists of many interconnected neuron elements. The network minimizes an energy function of the form

$$
E=\sum_{K=1}^{N} \sum_{l=1}^{N} T_{k l} V_{k} V_{l}-\sum_{k=1}^{N} I_{k} V_{k}
$$

where $\mathrm{N}$ is the number of neurons, $V_{k}$ is the output of kth neuron, $I_{k}$ is the bias term, and $T_{k l}$ is the interconnection weight between $\mathrm{kth}$ and 1 th neuron. The minimization is achieved by solving a set of motion equations satisfying

$$
\partial U_{i} / \partial t=-\partial E / \partial V_{i}
$$

where $U_{i}$ is the input of ith neuron. This HNN have been used in [6] for the segmentation of magnetic resonance images (MRI). The results have been acceptable, however the method have some limitations due to the stuck of the network in an early local minimum because the energy landscape in general has more than one local minimum due to the nonconvex nature of the energy surface. To overcome this problem, we have suggested in our previous work [7] some contributions, which can be summarized in the following main folds: 1 . Considering the minimization of the sum of the squared errors, leads to better minimization than the simple sum of errors, 2. A procedure for hill-climbing necessary to avoid entrapment in certain local minima with the result of being closer to the global minimum, and 3 . The minimization is achieved with a step function suitable to increase the convergence speed of the network within a prespecified period of time. Also, it was shown in [7] that the HNN is capable to give more accurate segmentation results as more as the increase of the pixels features given to the input of the network.

Similarly to the MRI images, the color images can be considered as multidimensional data as each pixel is represented by its three components in the RGB image planes. Furthermore, in the case of sputum cells color images, more information about the ROI, such as nucleus and cytoplasm regions, can be extracted based on some chromatic properties. Added to the features list of each pixel, these information can help for more accurate segmentation of the image to be considered for the analysis step for lung cancer diagnosis.

\section{Method}

To the input of HNN we have applied the RGB components of several sputum images. However, the extreme variations in the gray-levels of the images and the relative contrast among nuclei due to unavoidable staining variations among individual cells, the cytoplasm folds and the debris cells, make the segmentation less 
accurate and impossible its automatisation as the number of regions is difficult to be estimated in advance. On the other hand, the most important objective in processing cell clusters is the detection and accurate segmentation of the nuclei, because most quantitative procedures are based on measurements of nuclear features. For this reason, based on our collected database of sputum color images, we found an algorithm for NonSputum cell masking. Once these masked images are determined, they are given ,with some of the RGB components of the raw image, to the input of HNN. In the result section, we show the effectiveness of this preclassification step, in making a crisp segmentation of sputum color images.

\section{NonSputum Cell Masking Algorithm}

The Masking algorithm is derived from the difference in the brightness level in the RGB components of the sputum color image. Furthermore, the algorithm depends on the staining method by which the image is prepared. In our database of sputum color images, we have found two sorts of stained images. Some of the images are stained with blue-dye, resulting in dark-blue nuclei of all the cells present in the scene, and clear-blue cytoplasm, a sample of this sort is shown in Fig.1 (a). The other sort of images are stained with a close method to that of Papanicolaou, resulting in dark-blue nucleus of the small debris cells with their corresponding small clear-blue cytoplasm regions, and red sputum cell with dark-red nucleus and clear-red cytoplasm. Fig.3 (a) shows a sample of the second sort of sputum color images. Dealing with sputum color images of the first type described above, the nuclei pixels (included those of the debris cells) are detected in the $\mathrm{B}$ image plane with the following condition:

if $(B(x, y)<G(x, y))$, then $(B(x, y)=0)$

where $R(x, y), G(x, y)$ and $B(x, y)$ represent the pixel value in the $R G B$ image planes respectively. This mask is applied to the image of Fig.1(a) and the result is shown in Fig.1.(f).However, in case of the second type of images, the procedure to extract the sputum cells nuclei consists of the following steps:

1/ First, the debris cells are removed using the following mask:

if $(B(x, y)>G(x, y)$ or $B(x, y)>R(x, y))$, then $(B(x, y)=0)$

the result of this step applied to the sample of Fig. 3 is shown in Fig.3 (g).

$2 /$ To extract the sputum cells, first we remove the debris from the Red and Green intensity images using the result of the fist step. Then, to the RGB maskeddebris images we apply the following mask:

$$
\text { if }(\mathrm{R}(\mathrm{x}, \mathrm{y})<(\mathrm{G}(\mathrm{x}, \mathrm{y})+\theta)) \text {, then }(\mathrm{R}(\mathrm{x}, \mathrm{y})=0)
$$

the result of this step is shown in fig.3.(h).

3/ The nuclei of the sputum cells are extracted by the following mask:

$$
\text { if }((2 * \mathrm{G}(\mathrm{x}, \mathrm{y})+\theta)<(R(x, y)+B(x, y))) \text {, then }(\mathrm{G}(\mathrm{x}, \mathrm{y})=0)
$$

and the result is shown in fig.3 (i). The constant $\theta$ was fixed to 10 in proceeding all the cases.

\section{Segmentation Result \& Discussion}

We present results obtained with two sputum images with different complexity and also staining methods. Fig.1(a) shows a simple sputum color image with small dark 
blue nuclei of debris cells, and a big dark-blue nucleus of a sputum cell surrounded by its clear-blue cytoplasm region. Fig. I (b), (c) and (d) are respectively the corresponding component images in the RGB color space of the color image shown in Fig.1(a). The segmentation result shown in Fig. 1(e) is obtained using HNN introduced above, with the raw RGB components of the sample in Fig.1(a). This result is obtained with a class number fixed to 4 . Here all the nuclei are detected with their corresponding cytoplasm regions, however the background is represented by more than one class due to the variation of its pixels gray levels. Fig.1 ( $f$ ) shows the B component masked image obtained with the relation (3). All the nuclei are detected perfectly, however the cytoplasm pixels with those of the background are turned to zero values. Fig. $1(\mathrm{~g})$ shows the segmentation result obtained with class number fixed to 3 , using HNN by giving to its input the raw $R$ and $G$ components with the $B$ masked image shown in Fig.1 (f). The comparison between the two segmentation results, is given in Fig.2, which shows the energy function of the network when using two different input data. The curve $\mathbf{A}$ corresponds to the result shown in Fig. 1(e) and the curve B to the result in Fig. I(g). As can be seen, when using HNN with the masked image, the error is smaller than in the case when we applied all the RGB component images to the input of the network.

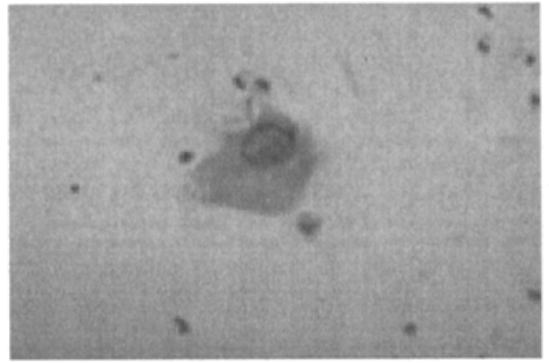

(a)

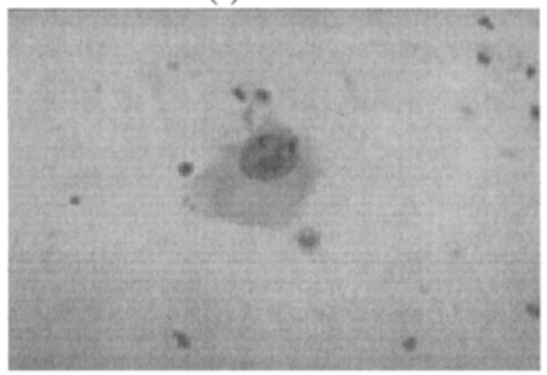

(c)

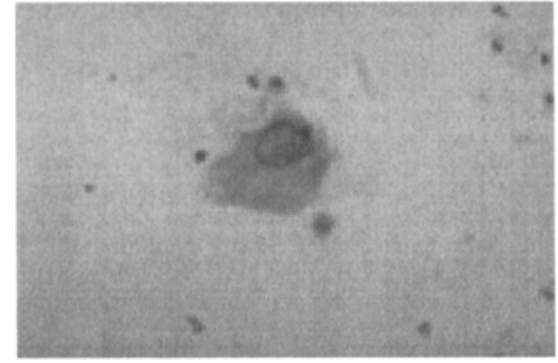

(b)

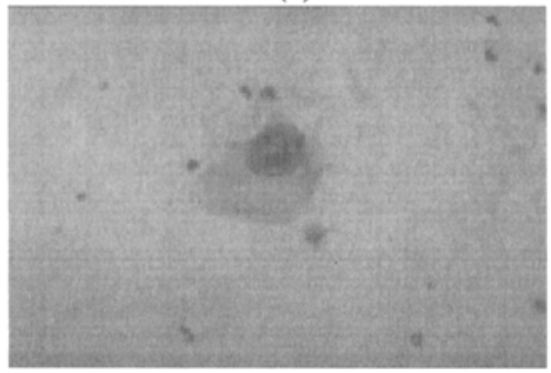

(d) 


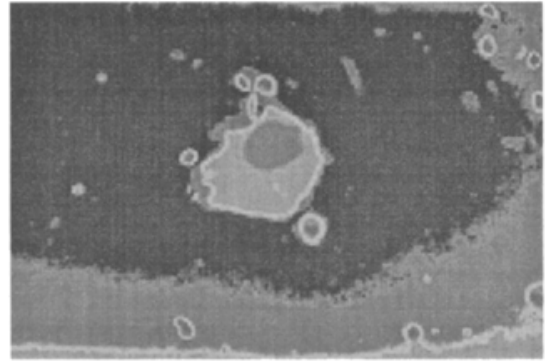

(e)

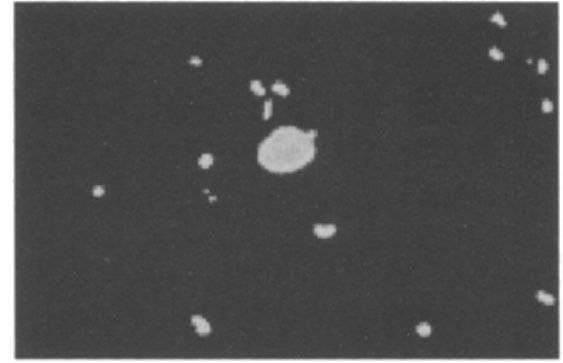

(f)

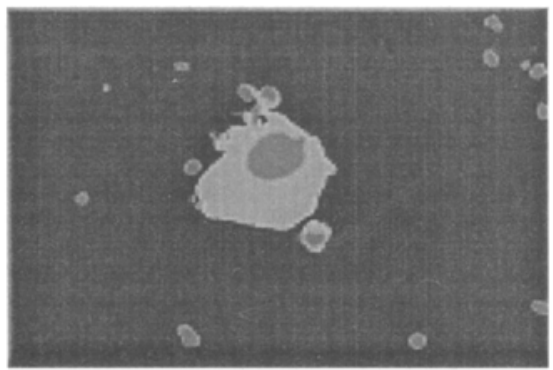

(g)

Fig.1. (a) shows a sample of color sputum image stained with a blue dye, (b), (c) and (d) are its components in the RGB image planes respectively. (e) is the segmentation result obtained using HNN without raw data, ( $f$ ) is B component masked image, and ( $g$ ) is the segmentation result obtained using HNN with preprocessed data .

Fig. 3. (a) shows a complex scene of sputum cells surrounded by a lot of debris nuclei and some variations in the gray levels of the background pixels. Fig.3 (b), (c) and (d) show the RGB components respectively. Fig. 3 (e) shows the segmentation result obtained using $H N N$ with the raw RGB components of the image as input data to the network. The network classifies most of the pixels in a good way, however it could not detect the nuclei of the big sputum cells, which are the most important for the diagnostic system. Fig. 3 (f) shows the debrismasked B component image obtained by applying the relation (4). By this mask all the small debris cells are removed. The same debris regions removed in the $B$ image are also removed in the $R$ and $G$ component images. To these debrismasked images we apply the condition (5), which result in detecting the sputum cells in the $G$ component image as shown in Fig. $3(\mathrm{~g})$. Also, we form the synonyms of this result with the $R$ and $B$ images, then we proceed these images using the condition (6) which result in detecting the nuclei of the sputum cells in the $\mathrm{G}$ component image as shown in Fig.3 (h). Fig.3 (i) is obtained using HNN, with 3 classes, by giving to its input the R and B images obtained by the relation (5) and the $G$ image obtained by the condition (6). Here, all the pixels are classified into a clear background, cytoplasm and nuclei. Fig. 4 shows the curves of the energy function of the network with the input data as described above. The curve $\mathbf{C}$ corresponds to the result shown in Fig.3(e) and the curve $\mathbf{D}$ to the result in Fig.3(i). So, the debris masking preprocessing helped a lot to let the network go more in the energy landscape to give a more accurate sputum cells segmentation. 


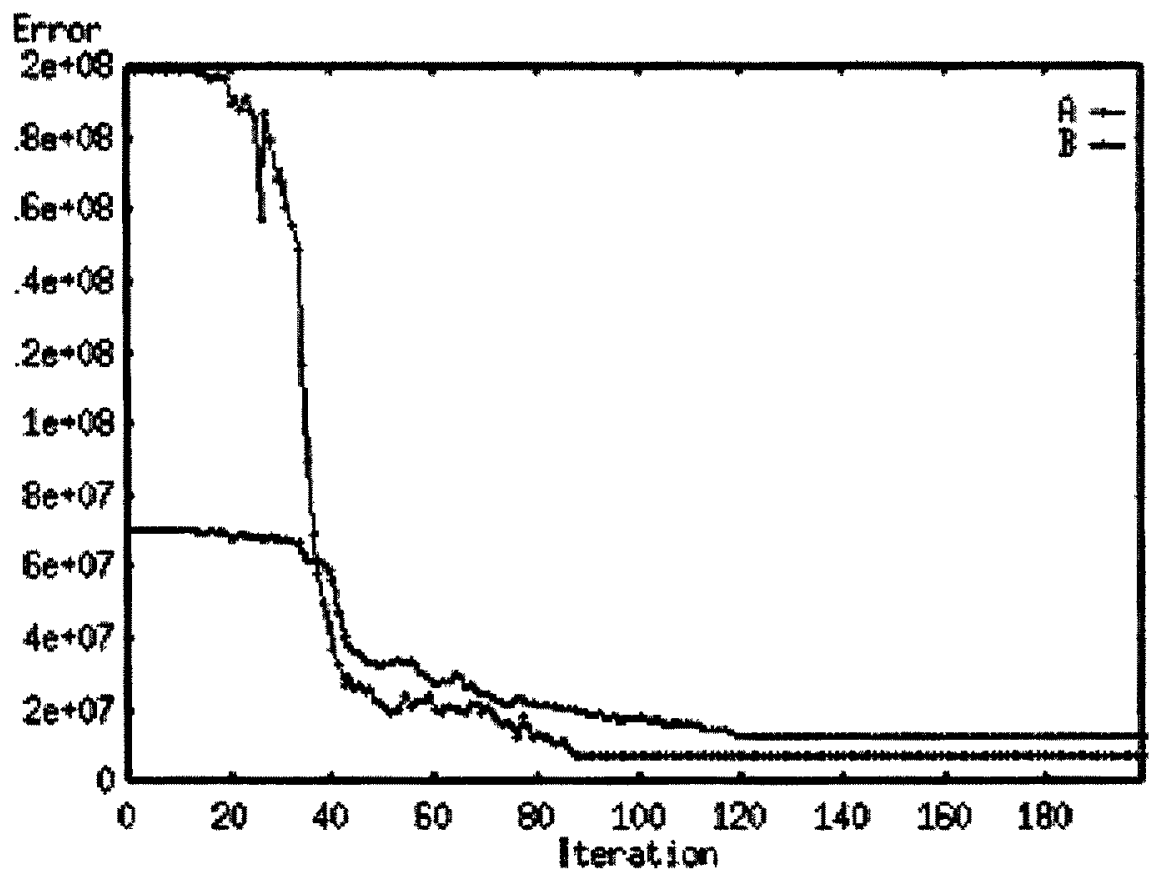

Fig.2. The curves A and B represent the energy function of the HNN during the optimization process of the sample shown in Fig. 1 with preprocessed data and raw data respectively.

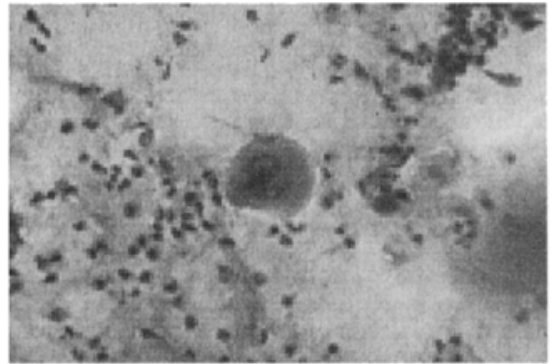

(a)

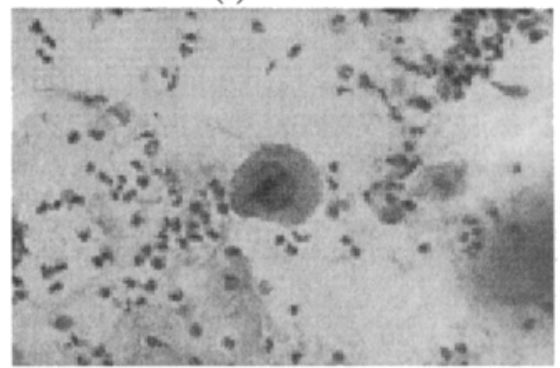

(c)

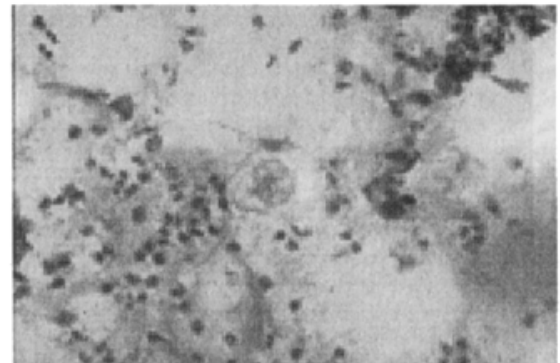

(b)

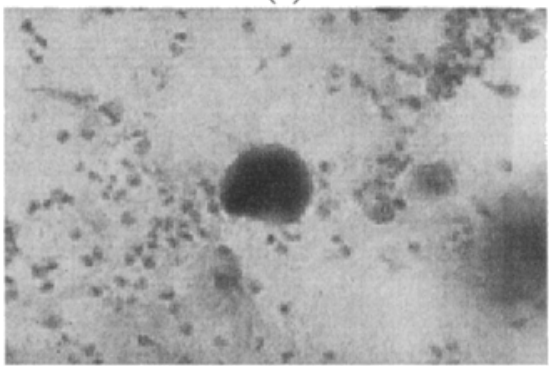

(d) 


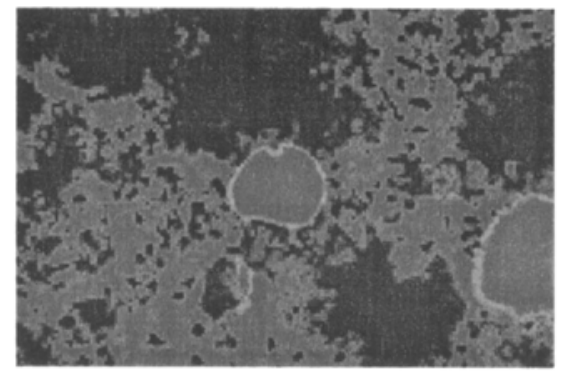

(e)

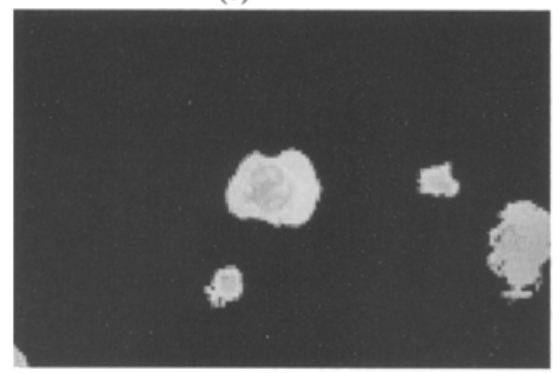

(g)

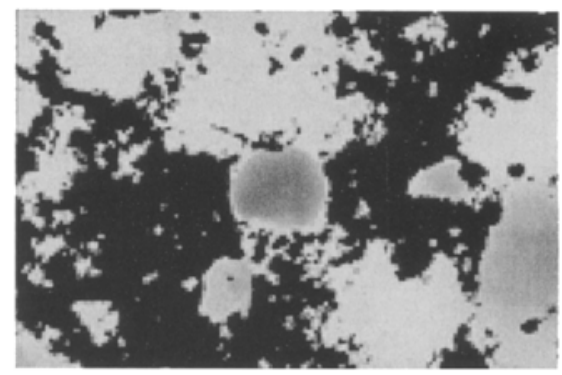

(f)

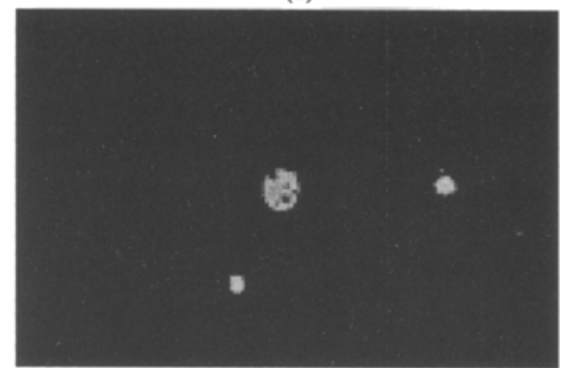

(h)

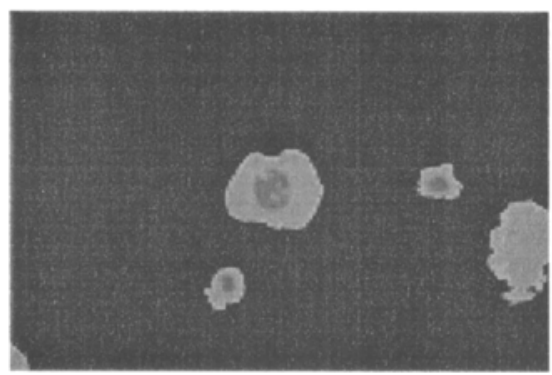

(i)

Fig.3. (a) shows a sample of sputum color image more complex than the previous one and stained with blue and red dyes. (b), (c) and (d) are the components of (a) in the RGB image planes respectively. (e) shows the segmentation result using HNN with raw components. (f) represents the debrismasked B component image. $(g)$ and (h) shows the extracted sputum cells image and their nuclei respectively obtained as described in the text. (i) is the segmentation results obtained using HNN with preprocessed data.

\section{Conclusion}

In this study, we presented a method for the segmentation of sputum cell color images, which will be considered into an analysis procedure for lung cancer diagnosis. The presented segmentation technique, uses a preclassification algorithm to extract the regions of interest within the raw color image components in the RGB color space, and remove the debris cells. As shown in the previous section, HNN could make a crisp classification of the cells, when proceeding the debrismasked images. However, in the case of overlapped cells, more work have to be done to remove the overlapping 
of the cells. Actually, we are looking to develop another preclassification procedure to detect and remove the overlapping between the cells. By doing this, the segmentation process can be automatic and more accurate to give results which can help for a successful lung cancer diagnosis.

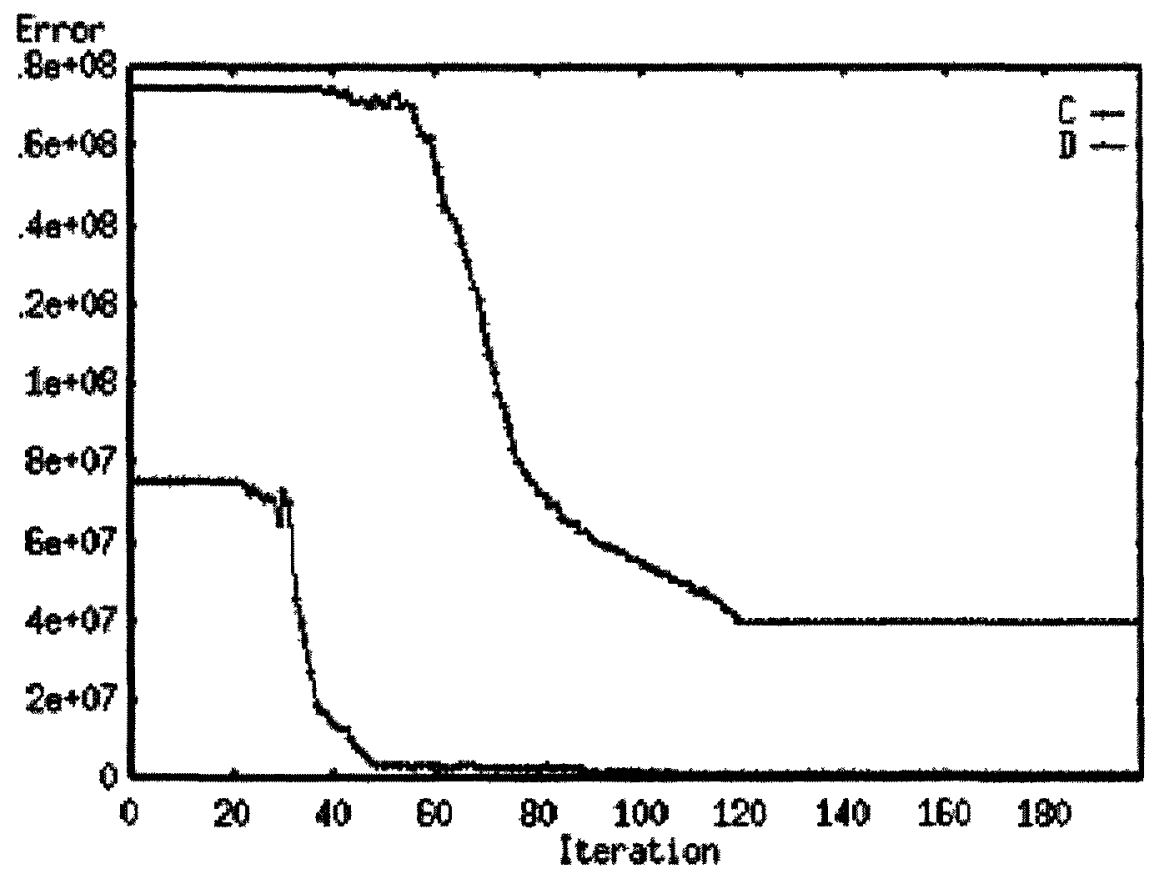

Fig.4. The curves $C$ and $D$ represent the energy function of the HNN during the optimization process of the sample shown in Fig. 3 with preprocessed data and raw data respectively.

\section{References}

1. J. Mayo, N. L. Muller, and R.M Henkelman "The double-fissure sign: A motion artifact on thin section CT scans", Radiology 165, 50-581, 1987.

2. R. D. Tarver, D. L. Conces, and J. D. Godwin, "Motion artifacts on CT simulate bronchiectasis" Am. J. Roentgenol. 151, 1117-1119, 1988.

3. Jiang Hsiel, "A genral approach to the reconstruction of the X-ray Helical Computed Tomography", Med. Phy. 23 (2), 221-229, February 1996.

4. Jian king Lin and Yee-Hong, "Multiresolution Color Image Segmentation", IEEE Transactions on Pattern Analysis and Machine Intelligence, vol. 16 No. 7, 689-700, July 1994.

5. Papanicalaou GN \& Trant HF, "Diagnosis of Uterine Cancer by the Vaginal Smear". Oxford University press, New York 1943.

6. S. C. Amartur, D. Piraino, and Y. Kakefuji, "Optimization Neural Networks for the Segmentation of Magnetic Resonance Images", IEEE Transaction on Medical Imaging, vol. 11, No. 2, June 1992.

7. R. Sammouda, N. Niki and H. Nishitani, "Segmentation of Brain MR Images Based on Neural Networks", IEICE Trans. Inf. \& Syst., vol. E79-D, No. 4, 349-356, April 1996. 